\title{
We Asked the Experts: Providing the Road Map to Uncovering the Pathophysiology of Young-Onset Cancer to Guide Treatment and Preventive Strategies
}

\author{
Savio George Barreto ${ }^{1,2}$
}

Published online: 14 June 2020

(C) Société Internationale de Chirurgie 2020

Until recently, the jury was still out on whether 'youngonset' cancer, defined as cancer occurring in individuals under the age of 40 years, was a distinct entity with outcomes worse off than sporadic cancers that generally occur in older individuals [1]. In the last 5 years, there have been numerous publications highlighting a rise in the incidence of young-onset cancers affecting the ovary, breast, rectum, and pancreas. Interestingly, the aetiology of these cancers cannot be traced back to pre-existing familial cancer syndromes [2, 3]. We are, thus, clearly beyond the stage of debating the existence of the entity. It is time to set the agenda for our approach to deciphering its pathophysiology to enable us develop strategies for early detection, appropriate treatment, and the identification of opportunities, if any, to prevent these cancers.

Thus far, the risk factors linked to the development of young-onset cancers appear similar to those involved in sporadic cancers, namely alcohol, smoking, and obesity. However, does our current understanding of carcinogenesis allow us to accept these findings at face value, or are we regressing to a 'one-size-fits-all' approach in oncology? A recent publication by Lahouel et al. [4] presented for the first time a data-driven, mathematical model of the process of tumour evolution taking into consideration the fitness advantages for driver genes and carrying capacity [4]. The authors used their vast experience over decades of cancer research to prepare the model that accounts for the three

Savio George Barreto

georgebarreto@yahoo.com; savio.barreto@sa.gov.au

1 Division of Surgery and Perioperative Medicine, Department of Surgery, Flinders Medical Centre, Bedford Park, Adelaide, SA 5042, Australia

2 College of Medicine and Public Health, Flinders University, Adelaide, SA, Australia mechanisms that confer fitness advantage, namely cell fate, cell survival, and genome maintenance, and total number of clonal somatic mutations accumulated in a cell lineage. What is striking in this manuscript is the discovery of the timing of driver gene mutations. The authors noted the first hit to occur at a median age of 14.4 years for colon cancer and 14.6 years for pancreatic cancer, with the full development of malignancy taking on average another 50 years. In young-onset cancers, we can postulate an acceleration of events in terms of the timing of both first and second 'hits'. It is difficult to accept that an individual would be exposed to the proposed risk factors within the first couple of decades of life in a magnitude to an extent that would induce mutations and cancer by the age of 40 years. I hypothesize that what is more likely to play a role in carcinogenesis in young-onset cancers is an 'in utero' insult to the foetus that speeds up, or leads to, the 'first hit'. The second hit would then be the result of processes occurring in childhood and adolescence. It would be worthwhile, at this point, to acknowledge the hypothesis referred to as the 'Developmental Origins of Health and Disease (DOHaD)' [5]. This hypothesis highlighted the significance of exposure of the developing foetus to a hostile (nutritional deprivation or excess, chemical exposure, or infections) environment in relation to the risk of diseases in adult life. These in utero insults can trigger epigenetic and hormonal modifications designed to permit the growing foetus to adapt and survive. However, it is likely that the effects of exposure to similar risk factors in early childhood and adolescence could result in a premature activation of driver mutations (the second hit) rendering the young adult 'at risk' of developing a cancer by a process referred to as 'stress-induced' mutagenesis. There are many potential candidates for the second hit. Some have hypothesized antibiotic usage and 
nutritional excess or deficiency, owing to their ability to alter the gut microbiome [2].

However, we cannot ignore an increasingly possible driver, childhood obesity. Over the last three to four decades, the incidence of overweight and obese children has risen phenomenally, in both developing and developed countries. At this time, these associations would be regarded as conjecture, or temporal, at best. However, the Cancer Generative Model [4] presents itself as a promising tool to investigate the sequence/or timing of driver events in carcinogenesis in young patients. With the availability of ever-increasing amount of data derived from genome-wide sequencing, the Cancer Generative Model [4] allows us the opportunity to test these hypotheses, thereby enabling us to clarify key events in young-onset carcinogenesis.

Once we are able to create a timeline for the carcinogenesis in young patients, we would then be in a position to identify opportunities for intervention from primary prevention strategies targeting pregnant mothers with risk factors (substance abuse, obesity, etc.) to secondary prevention aimed at 'at-risk' individuals to reduce their exposure to putative risk factors. The information generated from a better understanding of the timeline of carcinogenesis would also serve to guide International Collaborative Initiatives, such as the CONCORD programme [6], to suggest recommendations on cancer surveillance for young adults who are deemed 'at risk'

In terms of our current management algorithms for the use of chemo- and/or radiotherapy in cancer that are largely based on a 'one-size-fits-all' approach, once again there are sufficient clues to suggest that using the same regimens of treatment in the young and old, alike, does not translate into comparable outcomes. My colleagues and I have previously noted a significantly poorer survival for youngonset stage III rectal cancer patients compared to their older counterparts when adopting the same neoadjuvant chemo-radiotherapy and adjuvant chemotherapy protocols [1]. The literature is rife with similar examples in other young-onset cancers. There are also the issues of overuse of chemotherapy in the young without a proven survival benefit [7], and lack of consensus on safe dosage levels of radiation therapy in younger adults. Young-onset cancer patients thus represent a cohort for whom pharmacogenomic analyses are warranted to clarify what treatment regimens would be most efficacious for them. The hope is that providing precision-based, personalized medicine will translate into improved overall survival.

In conclusion, young-onset cancers are a distinct and real entity. I have highlighted opportunities to investigate this cohort of patients to reduce their overall incidence and improve survival.

\section{Compliance with ethical standards}

Conflict of interest The author declares that he has no conflict of interest.

\section{References}

1. Barreto SG, Chaubal GN, Talole S et al (2014) Rectal cancer in young Indians-are these cancers different compared to their older counterparts? Indian J Gastroenterol 33:146-150

2. Barreto SG (2019) Young-onset rectal cancer patients: in need of answers. Future Oncol 15:1053-1055

3. Bernards SS, Norquist BM, Harrell MI et al (2016) Genetic characterization of early onset ovarian carcinoma. Gynecol Oncol 140:221-225

4. Lahouel K, Younes L, Danilova L et al (2020) Revisiting the tumorigenesis timeline with a data-driven generative model. Proc Natl Acad Sci U S A 117:857-864

5. Barker DJ (2007) The origins of the developmental origins theory. J Intern Med 261:412-417

6. Bleich SN, Vercammen KA, Zatz LY et al (2018) Interventions to prevent global childhood overweight and obesity: a systematic review. Lancet Diabetes Endocrinol 6:332-346

7. Manjelievskaia J, Brown D, McGlynn KA et al (2017) Chemotherapy use and survival among young and middle-aged patients with colon cancer. JAMA Surg 152:452-459

Publisher's Note Springer Nature remains neutral with regard to jurisdictional claims in published maps and institutional affiliations. 